\title{
Correction to: A rare case of a giant arterio- venous fistula (AVF) following metastatic choriocarcinoma conditioning pulmonary embolism: multimodal transcatheter embolization using a simultaneous transarterial and transvenous approach
}

\author{
Massimo Venturini ${ }^{1 *}$, Alice Bergamini ${ }^{2}$, Anna Colarieti ${ }^{1}$, Micaela Petrone $^{2}$, Paolo Marra ${ }^{1}$, Emanuela Rabaiotti $^{2}$, \\ Giorgia Mangili' ${ }^{2}$, Massimo Candiani ${ }^{2,3}$, Alessandro Del Maschio ${ }^{1,3}$ and Francesco De Cobelli, ${ }^{1,3}$
}

Correction to: CVIR Endovasc (2018) 1:30

https://doi.org/10.1186/s42155-018-0039-8

'In the published article (Venturini et al. 2018) the statement under the subheading 'Consent for publication' is incorrect.

"Not applicable"

should read:

"Informed consent for publication of this case report and its accompanying images has been obtained from the patient"

\section{Consent for publication}

Informed consent for publication of this case report and its accompanying images has been obtained from the patient.

\section{Author details}

${ }^{1}$ Department of Radiology, San Raffaele Scientific Institute, Vita-Salute San Raffaele University, Via Olgettina 60, 20132 Milan, Italy. ${ }^{2}$ Department of Gynaecology, San Raffaele Scientific Institute, Milan, Italy. ${ }^{3}$ Vita-Salute San Raffaele University, Milan, Italy.
Published online: 02 March 2020

\section{Reference}

Venturini M, Bergamini A, Colarieti A, Petrone M, Marra P, Rabaiotti E, Mangili G, Candiani M, Del Maschio A, De Cobelli F (2018) A rare case of a giant arteriovenous fistula (AVF) following metastatic choriocarcinoma conditioning pulmonary embolism: multimodal transcatheter embolization using a simultaneous transarterial and transvenous approach. CVIR Endovasc 1:30. https://doi.org/10.1186/s42155-018-0039-8

The original article can be found online at https://doi.org/10.1186/s42155018-0039-8

*Correspondence: massimo.venturini@uninsubria.it

${ }^{1}$ Department of Radiology, San Raffaele Scientific Institute, Vita-Salute San

Raffaele University, Via Olgettina 60, 20132 Milan, Italy 